\title{
AFRONTAMIENTO ENFERMERO ANTE LA DESIGUALDAD Y EL RACISMO
}

\author{
Sergio Romeo López Alonso
}

Ldo. Enfermería. Master en Salud Pública. Hospital San Cecilio (Fecha de aceptación: 15/2/2002)

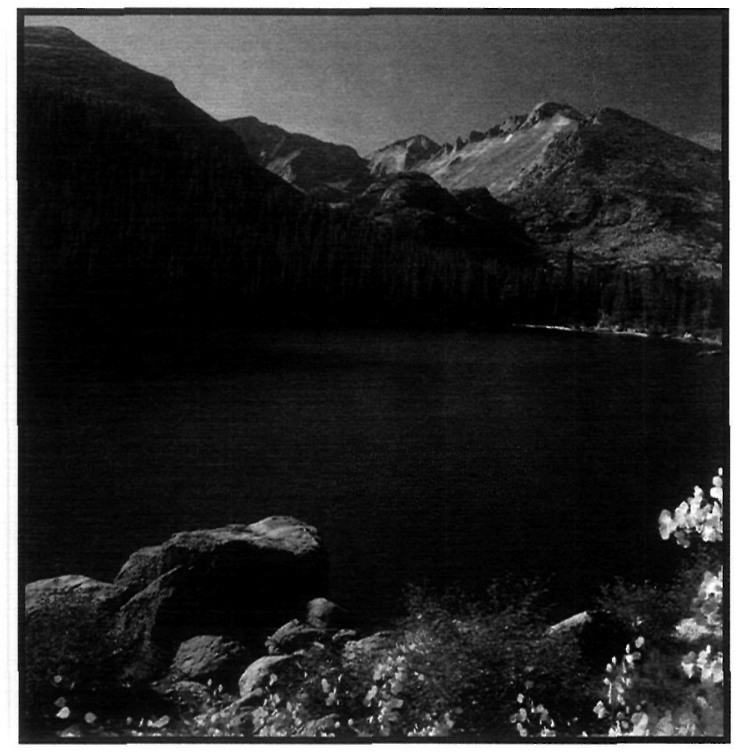

\section{NURSING COPING WITH INEQUITY AND RACISM}

\section{ABSTRACT}

7 he present text is introduced in the field of transcultural nursing, trying to approach the health problems appeared as a result of globalization. This approach is made from a multicultural position, where the curriculum development and the nursing model play important roles. Thus, the establishment of the nursing process will help tackling effectively the new health demands that are taking place in today's society.

Subject Headings: social class, racism, transcultural nursing, curriculum development.

\section{RESUMEN}

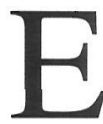

1 presente texto se introduce en el campo de la enfermería transcultural, tratando de abordar los problemas de salud aparecidos como consecuencia de la globalización. Este abordaje se realiza desde una posición multicultural, partiendo de la formación curricular y desnudando, posteriormente, un modelo enfermero para ponerlo a merced del proceso de atención de enfermería, con el fin de conseguir un afrontamiento efectivo sobre las nuevas demandas de salud que se están produciendo en la sociedad actual.

Descriptores: clase social, racismo, enfermería transcultural, currículum vitae.

\section{INTRODUCCIÓN}

ctualmente, el fuerte poder económico de
los países más desarrollados dirigido por
la política capitalista comienza a hacer mella en todas y cada una de las dimensiones del resto de las sociedades. Así, la creciente circulación de bienes y personas se manifiesta de forma destacada en la cultura, empleo y, consecuentemente, en la salud. El fenómeno de la globalización nos brinda una innumerable serie de ventajas como puede ser el intercambio de información, las tecnologías innovadoras, el reconocimiento de retos comunes sobre la salud pública, la conservación del medio ambiente, los derechos humanos, el sistema legal y otros. Sin embargo, y lamentablemente, esta misma política capitalista nos lleva a la polarización entre los países más y menos desarrollados a través de la explotación económica y el imperialismo cultural (Human Development report, 1999).

En este sentido, la inversión de la pirámide de población y el rechazo de los puestos de trabajo menos deseados, como la agricultura, hace que la emigración regulada de personas jóvenes sea bienvenida, ya que una adecuada entrada de individuos en los países desarrollados provee de fuerzas de trabajo para la explotación de los recursos nacionales, reduciendo la inflación y mejorando la capacidad de progreso económico (Stalker, 1997).

DESIGUALDAD Y RACISMO EN LA SALUD

Si bien la inmigración puede ofrecer grandes beneficios para el país de destino, es necesario 
cierta homogeneidad en el nivel de salud de estos nuevos ciudadanos equiparable al del resto de la población. De esta manera, se estará persiguiendo la equidad en salud con el objetivo de lograr el desarrollo sostenido y eficiente de otras áreas como el empleo, la educación y la economía.

Desgraciadamente, esta situación no es así. Existe una masiva entrada fuera de control de estos individuos, que produce desempleo e inevitablemente pobreza, delincuencia y explotación sexual. Todo esto, enmascara las contribuciones positivas de la emigración y potencian el prejuicio, los estereotipos y el racismo (López Alonso, 2001).

Por una parte, el acceso a los servicios de salud aparecen mermados por varios motivos. Entre ellos, la pobreza, la cultura, el bajo nivel de educativo y la barrera idiomática han sido calificados como los principales (Gerrish, 1999). Estos factores, no sólo reducen el acceso a los servicios de salud, sino que ya de por sí contribuyen directa o indirectamente al deterioro de la salud, creando determinados problemas y necesidades características de estos grupos.

Por otra parte, el prejuicio, los estereotipos y el racismo se ponen ineludiblemente de manifiesto, unas veces de manera consciente y otras inconsciente. En muchas ocasiones se produce en forma de negligencia. Así, se puede citar la falta de atención ante las necesidades específicas de la comunidad islámica, o el estereotipo de falta de instinto maternal encontrado en ciertas matronas de mujeres asiáticas (Bowler, 1993).

Todas estas situaciones hay que sumarlas a la falta de intervención para contrarrestar sus perjuicios, produciendo así un descenso en el control sobre las respuestas de estos individuos o familias ante la presencia de enfermedades y ante la exposición a factores de riesgo. Y consiguientemente, también desciende el control sobre sus patologías. Por tanto, existe una depreciación en el nivel de salud de estos individuos.

\section{AFRONTAMIENTO ENFERMERO DESDE UNA IDEOLOGÍA MULTICULTURAL}

Este enfoque ha sido muy trabajado dentro del colectivo enfermero. Desde esta posición, se proclama que la promoción de la salud de estos individuos es sensible a la comprensión y tolerancia hacia las diferentes culturas. Así, en los últimos años, ha aparecido un gran número de teorías transculturales con las que se pretende facilitar el proceso enfermero y alentar a aquellas personas que de una manera u otra experimentan desigualdades en su nivel de salud debido a sus diferencias culturales con el grueso de la población.

\section{Formación curricular}

Un estudio realizado por Rooda (1993) sobre el conocimiento y actitudes de las enfermeras en pacientes con culturas diferentes mostró que, de las múltiples variables que analizó, el nivel de formación educacional fue el más sensible a la calidad de los servicios prestados, y por tanto el más significativo.

Esta investigación suscita una mirada atenta a los objetivos, contenidos y experiencia práctica sobre la diversidad cultural, con el fin de preparar a los futuros profesionales para una, cada vez más cambiante, sociedad a merced de los efectos de la globalización.

En este caso, la educación curricular tiene como fin último la formación de profesionales de enfermería competentes y capaces de brindar una creciente calidad en el cuidado. De este modo, es importante reconocer otras culturas distintas a la propia, con el objetivo de contextualizar el cuidado desde la cultura del individuo y del profesional.

Así, y conforme a Abdullah (1995), los objetivos de la educación deben estar centrados en:

- El aumento del conocimiento, sensibilidad y compromiso transcultural. La individualización

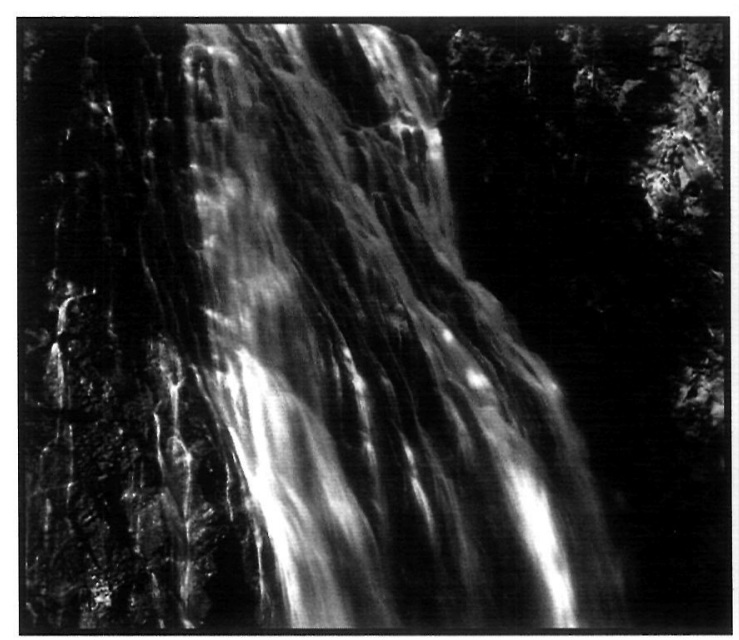


del cuidado vendrá determinada por los datos recogidos sobre variables que, como la religión, valores, dieta o creencias, influyen en los comportamientos de los individuos. El uso de modelos provee de una base conceptual a los profesionales de enfermería para fundamentar sus herramientas de trabajo, como el proceso enfermero o los planes de cuidados estandarizados.

- El incremento de conciencia sobre los diversos valores influyentes. Esta conciencia debe partir desde los valores filosóficos de la institución, y reflejarse en la provisión del cuidado. La enseñanza ha de ser guiada dentro de un paradigma enfermero, el cual debe reconocer el contexto cultural donde se imparte.

- La mejora de las habilidades de comunicación efectiva. Una comunicación defectuosa puede desentender información de gran relevancia en la valoración del individuo. Es por ello que los profesionales deben desarrollar este área para adentrarse en la experiencia que el propio individuo tiene sobre su situación de salud.

\section{Modelo enfermero}

El modelo enfermero ayuda a homogenizar los cuidados, ofreciendo un enfoque filosófico y describiendo los aspectos más importantes para la prestación de estos cuidados. En rasgos generales, los modelos son perspectivas diferentes desde donde se pretende la aproximación a un individuo, y éste sirve de base conceptual para la aplicación del proceso enfermero. De este modo, cualquier modelo nos será útil, aunque con ello no se afirme que los modelos sean universales. Habrá modelos que se adecuen mejor a las necesidades de determinados individuos $\mathrm{y}$, consiguientemente, a las repuestas humanas que de ellas se desprendan. Está en manos del profesional de enfermería el reconocer qué modelo se adapta mejor al individuo a tratar y a su forma de trabajo, con el fin de lograr los mejores resultados.

Entre los numerosos estudiosos de las teorías transculturales, puede citarse a Leininger como la más destacada. Madeleine Leininger (1995) nos insta a acrecentar nuestros conocimientos sobre los valores culturales y así llevar a cabo un pensamiento crítico para realizar un cuidado satisfactorio. De esta manera, pretende converger la práctica tradicional de cada individuo y la práctica de la enfermería en un cuidado culturalmente congruente. La valoración se realiza dentro la cultura, englobando aquí las distintas contingencias sociales y culturales, la etnohistoria y las creencias de salud. La naturaleza holística e integral de la teoría transcultural de Leininger hace que esta teoría presente numerosas interrelaciones entre la estructura social, el entorno y el lenguaje con los propios conceptos transculturales, y ello hace compleja su comprensión sin una formación juiciosa sobre su teoría y sobre conocimientos transculturales. Sin embargo, una vez lograda esta formación, la práctica es muy efectiva, tanto en la investigación y educación como en la práctica clínica (Marriner-Tomey, 1999). No obstante, se debe mencionar que no aporta ningún instrumento para superar los prejuicios y estereotipos de los propios enfermeros. Ciertamente, tal formación debe proporcionar una madurez cultural suficiente para traspasar cualquier variante de racismo por parte del profesional.

No obstante, y para aquellos enfermeros no tan adentrados en conocimientos culturales, se ha seleccionado otro modelo menos complejo, que si bien no ha sido comprobado científicamente, parece reunir las características necesarias para aportar una base conceptual de gran utilidad.

El modelo que se presenta a continuación ha sido creado por Papadopoulos et al (1998). Este modelo de desarrollo de habilidades transculturales está compuesto por cuatro conceptos fundamentales: conciencia, conocimiento, sensibilidad y competencia cultural.

La conciencia cultural pretende cuestionar nuestro propio sistema de valores y cómo se construye desde la sociedad. De este modo, la reflexión crítica facilitará el abordaje, desde diferentes perspectivas, del sistema de valores de los servicios sociales y de salud.

El conocimiento cultural responde a la comprensión de distintas culturas, los enlaces existentes entre ellas, principalmente en materia de salud. Este cuerpo de conocimientos debe extraerse desde la sociología y, posteriormente, desde el propio sujeto al cual van a ser proporcionados los cuidados, haciendo de éstos una atención individualizada. 
Se entenderá por sensibilidad cultural aquella disposición presentada hacia los individuos con una cultura diferente a la nuestra. Desarrollar habilidades de comunicación interpersonal ayudará a promover el respeto, la confianza y la aceptación.

Y la competencia cultural, que será la consecución de una práctica antidiscriminatoria.

Figura 1. Modelo de desarrollo de habilidades transculturales. (Papadapoulos, 1998)

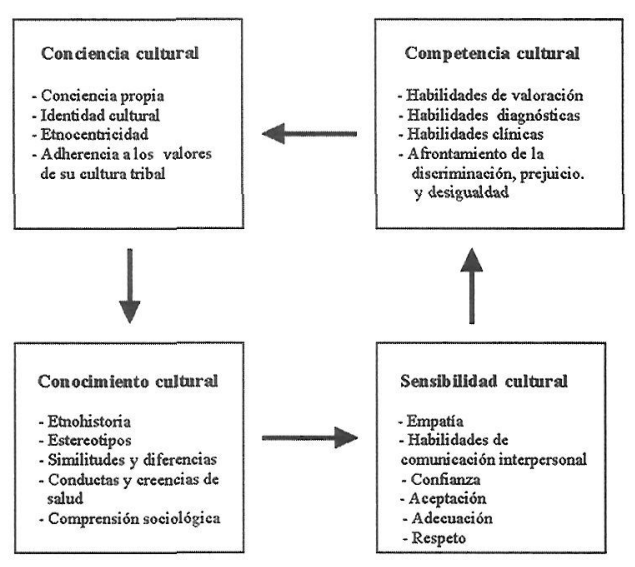

\section{Proceso enfermero}

El proceso enfermero proporciona las bases para el desarrollo del pensamiento crítico. Este proceso no es solamente un método para solucionar problemas, además previene su aparición, y maximiza el potencial y eficacia de la respuesta humana ante estos problemas, compensando las dificultades de la naturaleza humana, como pueden ser los sesgos de percepción. De esta forma, el uso del pensamiento crítico ayuda a realizar juicios fundamentados en la evidencia, basándose en los principios de la ciencia y del método científico para el logro de unos objetivos predeterminados (Alfaro-LeFevre, 1997). La contribución primordial del proceso enfermero será, por consiguiente, controlar la calidad de los cuidados.

\section{Valoración}

La valoración del individuo comienza en la valoración de la comunidad. Normalmente, los inmigrantes tienden a formar comunidades que conviven alrededor de un mismo área. La detec- ción de diferentes grupos étnicos en la población debe sugerir al profesional de enfermería a instaurar una reflexión crítica, tratando de tomar conciencia cultural, principalmente, de la identidad cultural como constructo social, de la adherencia a los valores tribales y de cómo estos afectan a la salud.

Una vez hecho esto, se debe identificar cuáles son los grupos étnicos minoritarios que forman parte de la comunidad. De este modo, habrá que tratar de formar un conocimiento cultural, e indagar en la etnohistoria de estos grupos. La sociología ayuda a conocer la situación de este grupo étnico en la población, a descubrir las similitudes y diferencias entre ambas culturas, y las creencias sobre la salud, así como los estereotipos más comunes. La comprensión sociológica de las características culturales de estos individuos, acercará a la perspectiva desde donde dan sentido a sus comportamientos y a la vida misma. Partiendo de aquí, la información recogida deberá ser verificada y matizada, contrastando los datos obtenidos con el sujeto objeto de nuestra atención con el fin de individualizar el cuidado.

A la sensibilidad cultural corresponde la empatía, la confianza y el respeto, por lo que se hace imprescindible el desarrollo de técnicas de comunicación interpersonal, como la escucha activa (León Rubio, 1997) y la relación de ayuda. Por otra parte, serán de gran apoyo las estrategias de comunicación que fomenten el pensamiento crítico, como las preguntas abiertas, y mostrar conductas que ayuden a construir relaciones interpersonales positivas (Alfaro-LeFevre, 1997).

El desarrollo de estos instrumentos aportará competencia cultural a la práctica enfermera. Es decir, se conseguirá reconocer las desigualdades y el racismo en los servicios de salud, y así lograr el desarrollo de habilidades de valoración, diagnóstico e intervención para afrontarlos de forma efectiva y generar una discriminación que conduzca a la equidad en salud.

\section{Diagnóstico enfermero}

En esta fase se pueden apreciar dos pasos (Luis Rodrigo, 1998):

A.- Análisis de los datos y la elaboración de inferencias e hipótesis. 


\section{Otros sistemas de apoyo: trabajadores bilin- gües}

Muchos son los estudios realizados sobre actividades que reduzcan las barreras en salud. Entre ellos, destaca por su efectividad la evidencia del empleo de trabajadores bilingües para contrarrestar las desventajas acaecidas por la desigualdad en el acceso a un servicio de salud de calidad.

Como ejemplo, se puede citar que la atención prestada por trabajadores bilingües mejora:

- El acceso a los servicios sociales (Mori Health, 1994). En este estudio, los resultados obtenidos fueron:

A. El $93 \%$ de usuarios de trabajadores bilingües, frente al 44 de no usuarios, manifestaban poder realizar preguntas a los médicos.

B. El $84 \%$ de usuarios, frente a un $39 \%$ de no usuarios, expresó que el médico le facilitaba explicaciones de sus enfermedades y tratamientos.

- La calidad de los servicios de salud (Rocheron, 1989). Este estudio suscitaba que el empleo de trabajadores con la misma identidad lingüística y cultural aumentaba la satisfacción de los individuos, y por tanto la calidad de los servicios prestados

- Los resultados clínicos. En estos estudios, la contratación de trabajadores bilingües brindaban los siguientes resultados:

A. Mejora significante de los niveles de hemoglobina y del peso al nacer. (City and Hackney Community Health Council, 1990)

B. Reducción del tiempo de estancia antes del parto y del número de cesáreas. (Parsons, 1992)

\section{DISCUSIÓN}

Desde la Declaración de Munich (II WHO Ministerial Conference on Nursing and Midwifery, 2000), la OMS decidió establecer como eje central de la salud en Europa a una enfermera de familia bien formada. Esto no se debe sólo al elevado número de estos profesionales, a su proximidad con la población y a la visión holística del cuidado de enfermería. Su fundamento se basa en cumplir con una función que únicamente la enfermera es capaz de desempeñar, el intervenir sobre las respuestas humanas ante situaciones de salud. De esta forma, los cuidados de enfermería permiten contribuir a la supervivencia, controlar y prevenir la morbilidad y sus secuelas, utilizar los recursos y las energías adecuadas, permitir la libre decisión del usuario, y mejorar la funcionalidad tanto del usuario como del sistema sanitario (Mompart García, 1995).

La nueva regulación de la sanidad pública (Ley orgánica 8/2000 de 22 de Diciembre) ha dado un paso al frente, otorgando a los inmigrantes el derecho a disfrutar de las ventajas que la sanidad pública ofrece a los ciudadanos. De este modo, se pretende homogeneizar el nivel de salud de la totalidad de la población, proporcionando oportunidades para promover los derechos y la prosperidad. Esta modificación legislativa se debe en parte a la búsqueda de un desarrollo sostenido en otras áreas, y está diseñada para afrontar de forma efectiva el imparable impacto de la globalización, tratando de maximizar sus beneficios.

Los profesionales de enfermería, como responsables de trazar la senda de la salud en la población, y como se precisó en la I Conferencia Ministerial sobre enfermería y matronas (I WHO Ministerial Conference on Nursing and Midwifery. Vienna Conference 1988), deben reforzar su status y aprovechar al máximo su enorme potencial para afrontar los retos públicos de salud de nuestro tiempo.

En este momento, el abordaje de la atención primaria sobre la población, aún en desarrollo en nuestro país, se está viendo desconcertado, ya que, de forma muy precipitada, una serie de necesidades específicas está apareciendo en la población, necesidades de las que hasta ahora sólo se conocían algunas pinceladas, pero que comienzan a formar parte de nuestro presente.

Por todo ello, se torna necesaria una intervención enfermera efectiva, que sea capaz de responder adecuadamente a las nuevas demandas que plantea la población. En este caso, la enfermería transcultural proporciona útiles para amortiguar las desigualdades en salud que, tristemente, sufren aquellas personas debido a sus características culturales.

\section{BIBLIOGRAFÍA}

- Abdullah, S.N. (1995) Towards an individualized client's care: implication for education. The transcultural approach. Journal of advanced nursing. 22(4): 715-720.

- Alfaro-LeFevre, R. (1997) El pensamiento crítico en enfermería. Un enfoque práctico. Masson, Barcelona. 
- Bowler, I. (1993) They're not the same as us: midwives'stereotypes of South Asian descent maternity patients. Sociology of Health and Illness. 15(2) 157- 178.

- City and Hackney Community Health Council. (1990) Experiments in Health Advocacy. London. City and Hackney $\mathrm{CHC}$.

- Gerrish, G. (1999) Inequalities in service provision: an examination of institutional influences on the provision of district nursing care to minority ethnic communities. Journal of advanced Nursing. 30:1263-1271.

- Human Development report. (1999) Oxford University Press: Oxford.

- Johnson, M., Maas, M. \& Moorhead S. (2000) Nursing Outcomes Classification. 2nd ed. St. Louis, Mosby.

- Leininger, M. (1995). Transcultural Nursing. Concepts, Theories, Research and Practices. 2nd Ed. McGraw Hill, New York.

- León Rubio, J.M. et al. (1997). Habilidades de información y comunicación en la atención al usuario. Junta de Andalucía, Universidad de Sevilla.

- Ley orgánica 8/2000 de 22 de Diciembre, de reforma de la ley orgánica 4/2000 de Enero, sobre derechos y libertades de los extranjeros en España y su integración social. Título 1, capítulo 1, artículo 12: Derecho a asistencia sanitaria.

- López Alonso, S. (2001) La globalización y la salud en los grupos minoritarios. Revista Cultura de los cuidados. $2^{\circ}$ semestre 2001. Año V - $\mathrm{N}^{\circ} 10$.

- Luis Rodrigo, M.T., Fernández Ferrín, C., Navarro Gómez, M.V. (1998), De la teoría a la práctica: El pensamiento de Virginia Henderson en el siglo XXI. Masson, Barcelona.

- Marriner-Tomey, A. (1999) Modelos y Teorías de Enfermería. $3^{a}$ Edición. Mosby/Doyma, Madrid.

McCloskey, J.C., Bulechek, G.M. (1999) Clasificación de Intervenciones de Enfermería. Editorial Síntesis, Madrid.

- Mompart García, M.P. (1995) Administración de servicios de enfermería. Masson: Barcelona

- Mori Health. (1994) Evaluation of Bilingual Health Care Schemes in East London. Mori, London.

- Papadopoulos, I., Tilky, M., Taylor, G. (1998) Transcultural care: A guide for health care professionals. Mark Allen Publishing, Trowbridge Wiltshire

- Parsons, L., Day, S. (1992) Improving obstetric outcomes in ethnic minorities: an evaluation of health Advocacy in Hackney. Journal of Public Health Medicine. 14 (2):183-191.

- Rocheron, V., Dickenson, R., Khan, S. (1989) Evaluation of the Asian Mother and Baby Campaign. University of Leicester.

- Rooda, L.A. (1993) Knowledge and attitudes of nurses towards culturally differnt patients: implications for nursing education. Journal of nursing education. 32(5):209-213.

- Stalker, P. (1997). Refugees and migration. 09 /05/2001

http://www.oneworld.org/guides/migration

- I WHO Ministerial Conference on Nursing and Midwifery. Vienna Conference 1988.

- II WHO Ministerial Conference on Nursing and Midwifery. Munich Declaration 2000: Nurses and midwifes-A force for health.

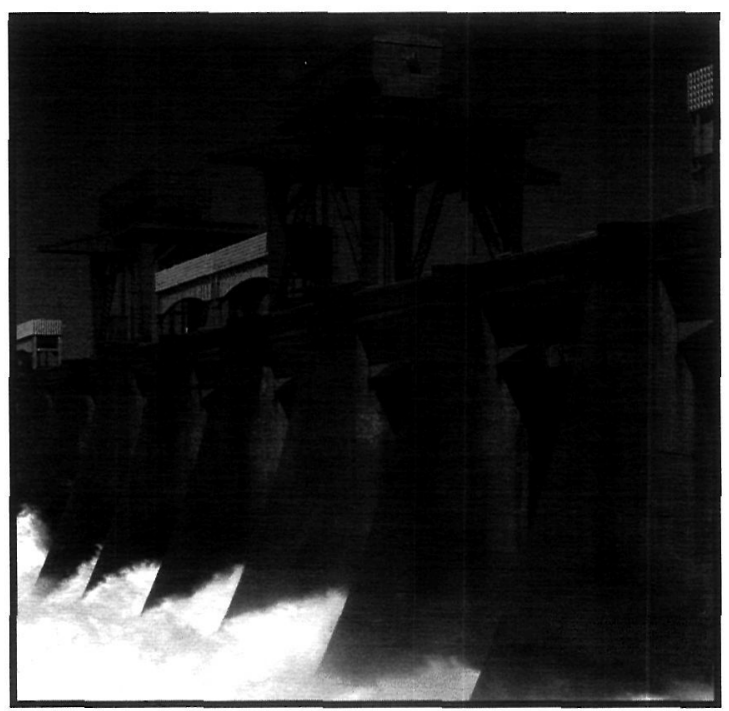

